\title{
Generation of Permutations Following Lehmer and Howell*
}

\author{
By Enrico Spoletini
}

\begin{abstract}
This paper proves two formulas used to obtain, by an arithmetic method, both the next permutation with respect to a given one and the $m$ th permutation, in lexicographic direct ordering.
\end{abstract}

1. Introduction. Almost all the methods used to generate permutations are based on exchanges and/or cycles and/or recursive techniques (see [4] and [5]).

Lehmer [3] developed a method to produce permutations of $k$ marks $0,1, \ldots, k-1$ in lexicographic ordering, based on the consideration that any permutation can be thought of as a base $k$ integer.

In [1] Howell improved the above result of Lehmer, showing that all permutations of the $k$ marks $0,1, \ldots, k-1$ may be obtained in the direct lexicographic ordering, from the permutation $01 \cdots(k-2)(k-1)$, regarded as a base $k$ integer, successively adding the number $k-1$ radix $k$, and rejecting all results which contain repeated digits.

In the present note this result is further improved upon, by computing the difference between two consecutive permutations in the direct lexicographic ordering. Moreover, an arithmetic method to find the $m$ th permutation (in the direct lexicographic ordering) is provided.

To avoid ambiguities in the following, we denote the $m$ th permutation by $P_{m}$ and the same permutation, considered as a base $k$ integer, by $\sigma_{k} P_{m}$.

2. Generation of the Next Permutation in the Direct Lexicographic Ordering. Let $P_{\alpha}$ be a permutation of the $k$ marks $0,1, \ldots, k-1$. $P_{\alpha}$ can be represented as a sequence $a_{k} a_{k-1} \cdots a_{2} a_{1}$ of $k$ numbers $a_{i}$ with $0 \leqslant a_{i} \leqslant k-1$ and $a_{i} \neq a_{s}$ for every $i \neq s(1 \leqslant i \leqslant k, 1 \leqslant s \leqslant k)$.

LEMMA 1. Let $a_{k} \cdots a_{h} \cdots a_{j} \cdots a_{1}$ be the permutation $P_{\alpha}$ of the $k$ marks $0,1, \ldots, k-1$ and suppose $a_{1}<\cdots<a_{j}<\cdots<a_{h-1} \nless a_{h}$ and $a_{j-1}<a_{h}<a_{j}$. Then $\sigma_{k} P_{\alpha+1}$ may be obtained by adding

$$
\Delta_{\alpha}=\sum_{i=1,[(h-1) / 2]} k^{i-1}\left(1-k^{h-2 i}\right)\left(a_{h-i}-a_{i}\right)+k^{h-j-1}\left(1-k^{j}\right)\left(a_{h}-a_{j}\right)
$$

radix $k$, to $\sigma_{k} P_{\alpha}$.

Received March 17, 1983; revised January 3, 1984.

1980 Mathematics Subject Classification. Primary 05Axx; Secondary 05A15.

Key words and phrases. Permutations, lexicographic ordering, lexicographic generation, permutation generation.

*This work is supported by Italian M.P.I. 
Proof. It is well known that, in our hypotheses, $P_{\alpha+1}$ may be obtained from $P_{\alpha}$ exchanging $a_{h}$ with $a_{j}$ and reversing the order of the $k$ marks $a_{h-1} \cdots a_{j+1} a_{h}$ $a_{j-1} \cdots a_{1}$ (see [5]). Then, computing $\Delta_{\alpha}=\sigma_{k} P_{\alpha+1}-\sigma_{k} P_{\alpha}$, we obtain

$$
\begin{aligned}
\Delta_{\alpha}= & \sum_{i=1, h-1} k^{i-1}\left(a_{h-i}-a_{i}\right)+k^{h-j-1}\left(a_{h}-a_{j}\right)+k^{h-1}\left(a_{j}-a_{h}\right) \\
= & \sum_{i=1,[(h-1) / 2]} k^{i-1}\left(a_{h-i}-a_{i}\right)+\sum_{i=1,[(h-1) / 2]} k^{h-i-1}\left(a_{i}-a_{h-i}\right) \\
& +\left(k^{h-j-1}-k^{h-1}\right)\left(a_{h}-a_{j}\right) \\
= & \sum_{i=1,[(h-1) / 2]} k^{i-1}\left(1-k^{h-2 i}\right)\left(a_{h-i}-a_{i}\right)+k^{h-j-1}\left(1-k^{j}\right)\left(a_{h}-a_{j}\right) .
\end{aligned}
$$

We wish to point out that, while the procedure described in [1] provides

$$
\sum_{i=1,[k / 2]} k^{i-1}(k-2 i+1)\left(k^{k-2 i}+k^{k-2 i-1}+\cdots+1\right)-k !+1
$$

integers which must be rejected in order to construct all permutations of the $k$ marks $0,1,2, \ldots, k-1$, by our method we obtain the only integers which are associated with some permutation.

Remark 2. By an analogous procedure, we may easily verify that, starting at the $\alpha$ th permutation $a_{k} \cdots a_{h} \cdots a_{j} \cdots a_{1}$ with $a_{1}>\cdots>a_{j}>\cdots>a_{h-1} \ngtr a_{h}$ and $a_{j-1}>a_{h}>a_{j}$, the previous permutation $P_{\alpha-1}$ may be obtained by adding to $\sigma_{k} P_{\alpha}$ the integer

$$
\Phi_{\alpha}=\sum_{i=1,[(h-1) / 2]} k^{i-1}\left(1-k^{h-2 i}\right)\left(a_{h-i}-a_{i}\right)+k^{h-j-1}\left(1-k^{j}\right)\left(a_{h}-a_{j}\right)
$$

radix $k$.

3. Generation of the $m$ th Permutation in the Direct Lexicographic Ordering.

TheOREM 3. Let $P_{1}$ be the permutation $012 \cdots(k-1)$ of the $k$ marks $0,1, \ldots$, $k-1$; let $m$ be an integer such that $m-1=\sum_{i=1, l} b_{i} i$ ! with $l<k$ and $0 \leqslant b_{i} \leqslant i$. Then the permutation $P_{m}$ may be obtained by adding, radix $k$, to $\sigma_{k} P_{1}$ the integer

$$
\begin{aligned}
\Delta^{m}= & \sum_{i=1, l}\left\{b_{i} k^{i}-\left(k^{i}-k^{i-b_{i}}\right) /(k-1)\right\} \\
& +\sum_{j=0, l-2} \sum_{i=j+1, l-1}\left\{\delta_{l-j, l-i}\left(k^{l-i}-k^{l-j-b_{l-j}-\sum_{h-l-i, l-j-1} \delta_{l-j, h}}\right)\right\}
\end{aligned}
$$

where $\delta_{v, v-1}=\delta\left(b_{v-1}-b_{v}+1\right)$ and, if $v-w>1$,

$$
\delta_{v, w}=\delta\left(v-b_{v}-\sum_{i=w+1, v-1} \delta_{v, i}-w+b_{w}\right) \quad \text { with } \delta(x)= \begin{cases}1 & \text { if } x>0, \\ 0 & \text { if } x \leqslant 0 .\end{cases}
$$

Proof. We will proceed by induction on $m$. For $m=1$ one has $\Delta^{1}=0$. Now suppose that the statement is true for $m$, some positive integer: we shall prove that it is true for $m+1$. To this end, in view of Lemma 1, it suffices to prove that

$$
\sigma_{k} P_{1}+\Delta^{m}+\Delta_{m}=\sigma_{k} P_{1}+\Delta^{m+1}
$$

or, equivalently,

$$
\Delta^{m+1}-\Delta^{m}=\Delta_{m}
$$


Let $m-1=\sum_{i=2, l} b_{i} i !+b_{1} 1 !(l<k)$ be the factorial representation of $m-1$. We shall distinguish two cases: $b_{1}=0$ and $b_{1}=1$.

Case 1. Let $b_{1}=0$. Then we have $m=\sum_{i=2, l} b_{i} i !+1$. Denoting by $\delta^{*}$ the $\delta$ 's computed for $\Delta^{m+1}$, it follows that $\delta_{i, j}=\delta_{i, j}^{*}$ for every $j>1$. Moreover, it is easily verifiable that

$$
\delta_{i, 1}^{*}=0 \quad \text { implies } \quad \delta_{i, 1}=0 \quad \text { and } \quad \delta_{i, 1}=1 \text { implies } \delta_{i, 1}^{*}=1
$$

and we have

$$
\delta_{i, 1}=0 \quad \text { and } \quad \delta_{i, 1}^{*}=1 \quad \text { if and only if } i-b_{i}-\sum_{j=2, i-1} \delta_{i, j}=1 .
$$

That being stated, we have

$$
\begin{aligned}
& \Delta^{m+1}-\Delta^{m}= \sum_{i=2, l}\left[\delta_{i, 1}^{*}\left(k-k^{i-b_{i}-\sum_{h=2, i-1} \delta_{i, h}-\delta_{i, 1}^{*}}\right)\right. \\
&\left.-\delta_{i, 1}\left(k-k^{i-b_{i}-\sum_{h=2, i-1} \delta_{i, h}-\delta_{i, 1}}\right)\right]+(k-1) \\
&=\sum_{i \in I}(k-1)+(k-1)=(1+|I|)(k-1),
\end{aligned}
$$

where $I=\left\{i \mid \delta_{i, 1}^{*}=1\right.$ and $\left.\delta_{i, 1}=0\right\}$, i.e., $I=\left\{i \mid i-b_{i}-\sum_{j=2, i-1} \delta_{i, j}=1\right\}$.

We remark that for $b_{1}=0$ in the $m$ th permutation $a_{k} \cdots a_{2} a_{1}$ we have $a_{2}<a_{1}$; therefore the $(m+1)$ st permutation may be obtained by adding to $\sigma_{k} P_{m}$ the integer $\left(a_{2}-a_{1}\right)(1-k)$, radix $k$ (Lemma 1). Then, it will suffice to verify that

$$
a_{1}-a_{2}=1+|I| \text {. }
$$

To this end we note that $a_{2}$ and $a_{1}$, by the action of $\Delta^{m}$, are respectively:

$$
\begin{array}{ll}
a_{2}=k-2+|N|-|M| & \text { where } N=\left\{i \mid \delta_{i, 1}=1\right\} \text { and } \\
& M=\left\{i \mid i-b_{i}-\sum_{h=1, i-1} \delta_{i, h} \leqslant 1\right\}, \\
a_{1}=k-1-|L| & \text { where } L=\left\{i \mid i-b_{i}-\sum_{h=1, i-1} \delta_{i, h}=0\right\} .
\end{array}
$$

Thus $a_{1}-a_{2}=1-|L|+|M|-|N|$. That being stated, let us show that $\delta_{i, 1}=1$ implies $i-b_{i}-\sum_{h=1, i-1} \delta_{i, h} \leqslant 1$. In fact, let us suppose

$$
i-b_{i}-\sum_{h=1, i-1} \delta_{i, h} \geqslant 2 \text { and } \delta_{i, 1}=1 \text {. }
$$

Then $\delta_{i, 1}=\delta\left(i-b_{i}-\sum_{h=2, i-1} \delta_{i, h}-1\right)=1$ and, by (1), it results that $i-b-$ $\sum_{h=2, i-1} \delta_{i, h} \geqslant 3$. Hence $\delta_{i, 2}=1$ and $i-b_{i}-\sum_{h=3, i-1} \delta_{i, h} \geqslant 4$. At this point it is clear that $\delta_{i, j}=1$ for every $j<i$. Replacing this result in the first relation of (1), we obtain the contradiction $i-b_{i}-i+1 \geqslant 2$. Then it follows that

$$
|M|-|N|=\mid\left\{i \mid i-b_{i}-\sum_{h=1, i-1} \delta_{i, h} \leqslant 1 \text { and } \delta_{i, 1}=0\right\} \mid \text {. }
$$

Moreover, from $i-b_{i}-\sum_{h=1, i-1} \delta_{i, h}=0$ it follows that $\delta_{i, 1}=0$; thus $|M|-|N|-$ $|L|=|I|$ where $I=\left\{i \mid i-b_{i}-\sum_{h=1, i-1} \delta_{i, h}=1\right.$ and $\left.\delta_{i, 1}=0\right\}$, i.e., $I=\left\{i \mid i-b_{i}-\right.$ $\left.\sum_{h=2, i-1} \delta_{i, h}=1\right\}$. Thus, in this case the statement is proved.

Case 2. Let $b_{1}=1$. Then we have $m=\sum_{i=r+1, l} b_{i} i !+\left(b_{r}+1\right) r$ !, where $r$ is the least positive integer such that $b_{r}<r$. Then it follows that $r \geqslant 2$ and $b_{r-i}=r-i$ for 
every $i$ with $0<i<r$. As in the preceding case, we shall denote by $\delta^{*}$ the $\delta$ 's computed for $\Delta^{m+1}$. We state now some propositions which shall be used in the sequel, and which may be deduced by examining the $\delta_{i, j}$ 's and $\delta_{i, j}^{*}$ 's.

$$
\begin{aligned}
& \text { If } j>r \text {, then } \delta_{i, j}=\delta_{i, j}^{*} . \\
& \delta_{i, r}^{*}=0 \rightarrow \delta_{i, r}=0, \quad \delta_{i, r}=1 \rightarrow \delta_{i, r}^{*}=1, \\
& \delta_{i, r}^{*}=1 \text { and } \delta_{i, r}=0 \Leftrightarrow i-b_{i}-\sum_{h=r+1, i-1} \delta_{i, h}-r+b_{r}=0 .
\end{aligned}
$$

If $i>r$, then, for every $j$ with $1 \leqslant j<r$, we have

$\delta_{i, r-1}^{*}=1 \rightarrow \delta_{i, r-j}^{*}=1$ and $\delta_{i, r-j}=1$,

$\delta_{i, r-1}=0 \rightarrow \delta_{i, r-j}=0$ and $\delta_{i, r-j}^{*}=0$.

If $i>r$ and $j<r$ then it follows that, for every $s$ with $j<s<r$, $\delta_{i, j}=1 \rightarrow \delta_{i, s}=1, \quad \delta_{i, j}^{*}=0 \rightarrow \delta_{i, s}^{*}=0$.

$\delta_{r, j}=1 \Leftrightarrow j \geqslant b_{r}, \quad \delta_{r, j}^{*}=1 \Leftrightarrow j<r-b_{r}-1$.

If $i<r$, then we have $\delta_{i, j}=0$ and $\delta_{i, j}^{*}=1$.

$\delta_{i, r}=1$ implies $\delta_{i, r-1}=1$.

$\delta_{i, r-1}^{*}=1$ implies $\delta_{i, r}^{*}=1$.

Let $i>r$. If there exists an integer $j<r$ such that $\delta_{i, j}=1$ $\left(\delta_{i, j}^{*}=0\right)$, then, by proposition (5), there is an integer $j_{i}\left(\hat{j}_{i}\right)$ so that $\delta_{i, j}=1\left(\delta_{i, j}^{*}=0\right)$ for every $j$ with $j_{i} \leqslant j<r\left(\hat{j}_{i} \leqslant j<r\right)$, while we have $\delta_{i, j}=0\left(\delta_{i, j}^{*}=1\right)$ for every $j$ with $0<j<j_{i}$ $\left(0<j<\hat{j}_{i}\right)$. Moreover, we obtain $i-b_{i}-\sum_{j=r, i-1} \delta_{i, j}=$ $r-j_{i}\left(i-b_{i}-\sum_{j=r, i-1} \delta_{i, j}^{*}=\hat{j}_{i}\right)$.

That being stated, let us compute $\Delta^{m+1}-\Delta^{m}$. In view of (2) and (7), since (7) implies $i-\sum_{h=j, i-1} \delta_{i, h}^{*}=j$ for every $i$ with $2 \leqslant i<r$, we have

$$
\begin{aligned}
& \Delta^{m+1}-\Delta^{m}=\left\{\sum_{i=1, r-1}\left[(i-r) k^{r-i}+k^{r-i-1}+\cdots+1\right]\right\} \\
& +\left\{k^{r}-k^{r-b_{r}-1}+\sum_{j=1, r-1}\left[\delta_{r, j}^{*}\left(k^{j}-k^{r-b_{r}-1-\Sigma_{h-j, r-1} \delta_{r, h}^{*}}\right)\right.\right. \\
& \left.\left.-\delta_{r, j}\left(k^{j}-k^{r-b_{r}-\sum_{h-j, r-1} \delta_{r, h}}\right)\right]\right\} \\
& +\left\{\sum _ { h = 1 , l - r } \left[\delta_{r+h, r}^{*}\left(k^{r}-k^{r+h-b_{r+h}-\sum_{i=r, r+h-1} \delta_{r+h, i}^{*}}\right)\right.\right. \\
& \left.\left.-\delta_{r+h, r}\left(k^{r}-k^{r+h-b_{r+h}-\sum_{i=r, r+h-1} \delta_{r+h, i}}\right)\right]\right\} \\
& +\left\{\sum _ { i = 1 , l - r } \sum _ { j = 1 , r - 1 } \left[\delta_{r+h, j}^{*}\left(k^{j}-k^{r+h-b_{r+h}-\sum_{i-j, r+h-1} \delta_{r+h, i}^{*}}\right)\right.\right. \\
& \left.\left.-\delta_{r+h, j}\left(k^{j}-k^{r+h-b_{r+h}-\sum_{i=j, r+h-1} \delta_{r+h, i}}\right)\right]\right\} .
\end{aligned}
$$

In view of (6), the second term of sum (11) becomes

$$
k^{r}-\sum_{j=b_{r}, r-1} k^{j}+\sum_{j=0, r-b_{r}-2} k^{j} .
$$


Now, we shall examine the third term; if $\delta_{r+h, r}^{*}=\delta_{r+h, r}$, making use of the proposition (2), we obtain

$$
\begin{aligned}
& \delta_{r+h, r}^{*}\left(k^{r}-k^{r+h-b_{r+h}-\sum_{i=r+1, r+h-1} \delta_{r+h, i}-\delta_{r+h}^{*}}\right) \\
& \quad-\delta_{r+h, r}\left(k^{r}-k^{r+h-b_{r+h}-\sum_{i=r+1, r+h-1} \delta_{r+h, i}-\delta_{r+h, r}}\right)=0 .
\end{aligned}
$$

If, on the contrary, we have $\delta_{r+h, r}^{*} \neq \delta_{r+h, r}$, then, by the third relation of proposition (3), we have $\delta_{r+h, r}^{*}=1$ and $\delta_{r+h, r}=0$, or equivalently,

$$
r+h-b_{r+h}-\sum_{i=r+1, r+h-1} \delta_{r+h, i}-r+b_{r}=0 .
$$

That being stated, the third term of the sum (11) becomes

$$
|M|\left(k^{r}-k^{r-b_{r}-1}\right),
$$

where $M$ is the set of the $h$ indices for which the equality (13) holds. Now, let us consider the fourth term of the sum and start by examining

$$
\begin{aligned}
\sum_{j=1, r-1}[ & \delta_{r+h, j}^{*}\left(k^{j}-k^{r+h-b_{r+h}-\sum_{i=r+1, r+h-1} \delta_{r+h, i}-\sum_{i=j, r} \delta_{r+h, i}^{*}}\right) \\
& \left.-\delta_{r+h, j}\left(k^{j}-k^{r+h-b_{r+h}-\sum_{i=r+1, r+h-1} \delta_{r+h, i}-\sum_{i=j, r} \delta_{r+h, i}}\right)\right] .
\end{aligned}
$$

If $\delta_{r+h, r-1}^{*}=1$, from (4) and (9) we obtain $\delta_{r+h, r-j}^{*}=1, \delta_{r+h, r-j}=1$ and $\delta_{r+h, r}^{*}=1$. Thus the term (15) is zero. If $\delta_{r+h, r-1}=0$, making use of (4), we have $\delta_{r+h, r-j}^{*}=0$ and $\delta_{r+h, r-j}=0$, and (15) is again zero.

It now remains to examine the case $\delta_{r+h, r-1}^{*}=0$ and $\delta_{r+h, r-1}=1$. By (10), denoting by $i_{r+h}$ and $\hat{i}_{r+h}$ respectively the least positive integers such that $\delta_{r+h, i_{r+h}}=1$ and $\delta_{r+h, \hat{i}_{r+h}}^{*}=0,(15)$ becomes, in view of (5),

$$
\begin{aligned}
& \sum_{j=1, \hat{i}_{r+h}-1}\left(k^{j}-k^{\left.\hat{i}_{r+h}-\sum_{i-j, i_{r+h^{-1}} \delta_{r+h, i}^{*}}\right)}\right. \\
& \quad-\sum_{j=i_{r+h}, r-1}\left(k^{j}-k^{r-i_{r+h}-\sum_{i=j, r-1} \delta_{r+h, i}}\right)=\sum_{j=i_{r+h}, r-1}\left(k^{j-i_{r+h}}-k^{j}\right) .
\end{aligned}
$$

Then the fourth term of the sum becomes

$$
\sum_{h \in H} \sum_{j=i_{r+h}, r-1}\left(k^{j-i_{r+h}}-k^{j}\right)
$$

where $H=\left\{h \mid 1 \leqslant h \leqslant l-r, \delta_{r-h, r-1}^{*}=0\right.$ and $\left.\delta_{r+h, r-1}=1\right\}$. In conclusion, from (11), (12), (14) and (16) it follows that

$$
\begin{aligned}
\Delta^{m+1}-\Delta^{m}= & \sum_{i=1, r-1}\left[(i-r) k^{r-i}+k^{r-i-1}+\cdots+1\right]+k^{r}-\sum_{j=b_{r}, r-1} k^{j} \\
& +\sum_{j=0, r-b_{r}-2} k^{j}+|M|\left(k^{r}-k^{r-b_{r}-1}\right) \\
& +\sum_{h \in H} \sum_{j=i_{r+h}, r-1}\left(k^{j-i_{r+h}}-k^{j}\right),
\end{aligned}
$$

that is,

$$
\Delta^{m+1}-\Delta^{m}=\sum_{i=1, r+1} c_{i} k^{i-1}
$$


where

$$
\begin{aligned}
& c_{i}=r-2 i+1+\delta\left(r-b_{r}-i\right)-\delta\left(i-b_{r}\right) \\
& \quad+\left|H_{i-1}\right|-\left|\bar{H}_{i-1}\right| \quad \text { if } i \neq r-b_{r}, i \leqslant r, \\
& c_{r+1}=1+|M|, \\
& c_{r-b_{r}}=2 b_{r}-r+1-\delta\left(r-2 b_{r}\right)+\left|H_{r-b_{r}}\right|-\left|\bar{H}_{r-b_{r}}\right|-|M|,
\end{aligned}
$$

and $H_{j}=\left\{h \in H \mid j \leqslant r-1-i_{r+h}\right\}, \bar{H}_{j}=\left\{h \in H \mid j \geqslant i_{r+h}\right\}$. Note that for $j=0$ it follows that $H_{0}=H$ and $\bar{H}_{0}=\varnothing$.

That being stated, let us remark that $m=\sum_{i=r+1, l} b_{i} i !+\left(b_{r}+1\right) r !$, and therefore for the last $r+1$ digits of the $(m+1)$ st permutation we have the relations

$$
a_{1}>a_{2}>\cdots>a_{r} \ngtr a_{r+1} \text { and } a_{r-b_{r}}<a_{r+1}<a_{r-b_{r}-1} .
$$

Then, the $m$ th permutation becomes $\alpha_{k} \cdots \alpha_{r+1} \alpha_{r} \cdots \alpha_{1}$ with $\alpha_{i}=a_{i}($ if $i>r+1)$, $\alpha_{r+1}=a_{r-b_{r}}, \alpha_{r}=a_{1}, \ldots, \alpha_{b_{r}}=a_{r-b_{r}+1}, \alpha_{b_{r}+1}=a_{r+1}, \ldots, \alpha_{1}=a_{r}$. Therefore we have $\alpha_{1}<\alpha_{2}<\cdots<\alpha_{r} \nless \alpha_{r+1}$ and $\alpha_{b_{r}}<\alpha_{r+1}<\alpha_{b_{r}+1}$. Then, by Lemma 1, we find

$$
\Delta_{m}=\sum_{i=1, r} k^{i-1}\left(\alpha_{r+1-i}-\alpha_{i}\right)+k^{r-b_{r}-1}\left(\alpha_{r+1}-\alpha_{b_{r}+1}\right)+k^{r}\left(\alpha_{b_{r}+1}-\alpha_{r+1}\right) .
$$

Hence, putting $L_{i}=\left\{h \mid h>0, \delta_{r+h, i}=1\right\}$ and

$$
N_{i}=\left\{h \mid h>0, r+h-b_{r+h}-\sum_{j=1, r+h-1} \delta_{r+h, j} \leqslant i\right\},
$$

we obtain

$$
\begin{aligned}
& \alpha_{r+1}=k-r-1+b_{r}+\left|L_{r}\right|-\left|N_{r}\right|, \\
& \alpha_{b_{r}+1}=k-r+b_{r}+\left|L_{b_{r}}\right|-\left|N_{b_{r}}\right| \quad \text { if } b_{r} \neq 0, \\
& \alpha_{i}=k-r+i-2+\left|L_{i-1}\right|-\left|N_{i-1}\right|+\delta_{r, i-1} \quad \text { if } 1<i \leqslant r, \\
& \alpha_{1}=k-1-r-\left|N_{0}\right| \quad \text { if } b_{r} \neq 0, \\
& \alpha_{1}=k-r-\left|N_{0}\right| \text { if } b_{r}=0 .
\end{aligned}
$$

That being stated, if $b_{r} \neq 0$, we have

$$
\alpha_{b_{r}+1}-\alpha_{r+1}=1+\left|L_{b_{r}}\right|-\left|L_{r}\right|+\left|N_{r}\right|-\left|N_{b_{r}}\right| .
$$

Making use of (8) and (10), it is easily shown that $\delta_{r+h, r}=1$ implies $\delta_{r+h, b_{r}}=1$, whence

$$
\left|L_{b_{r}}\right|-\left|L_{r}\right|=\mid\left\{h \mid h>0, \delta_{r+h, b_{r}}=1 \text { and } \delta_{r+h, r}=0\right\} \mid \text {. }
$$

From $\delta_{r+h, b_{r}}=1$, in view of (5), it follows that

$$
r+h-b_{r+h}-\sum_{i=r+1, r+h-1} \delta_{r+h, i}-\delta_{r+h, r}-r+b_{r}+1>0 .
$$

Moreover, $\delta_{r+h, r}=0$ is equivalent to

$$
r+h-b_{r+h}-\sum_{i=r+1, r+h-1} \delta_{r+h, i}-r+b_{r} \leqslant 0 .
$$


Hence, (18) and (19) hold together if and only if

$$
r+h-b_{r+h}-\sum_{i=r+1, r+h-1} \delta_{r+h, i}=r-b_{r}
$$

whence $\left|L_{b_{r}}\right|-\left|L_{r}\right|=|M|$. Moreover it is easily verifiable that

$$
\left|N_{r}\right|-\left|N_{b_{r}}\right|=\left|\left\{h \mid h>0, b_{r}<r+h-b_{r+h}-\sum_{i=1, r+h-1} \delta_{r+h, i} \leqslant r\right\}\right|=0 .
$$

Hence $\alpha_{b_{r}+1}-\alpha_{r+1}=1+|M|$. If $b_{r}=0$, we have

$$
\alpha_{b_{r}+1}-\alpha_{r+1}=\alpha_{1}-\alpha_{r+1}=1-\left|L_{r}\right|+\left|N_{r}\right|-\left|N_{0}\right| \text {. }
$$

After remarking that $r+h-b_{r+h}-\sum_{i=r+1, r+h-1} \delta_{r+h, i}>r$ implies $r+h-b_{r+h}$ $-\sum_{i=1, r+h-1} \delta_{r+h, i} \leqslant r$, it is easy to verify that $\left|N_{r}\right|-\left|L_{r}\right|-\left|N_{0}\right|=|M|$, whence we obtain again $\alpha_{b_{r}+1}-\alpha_{r+1}=1+|M|$. Let $1<i<r$, and consider

$$
\alpha_{r+1-i}-\alpha_{i}=r-2 i+1+\left|L_{r-i}\right|-\left|L_{i-1}\right|+\left|N_{i-1}\right|-\left|N_{r-i}\right|+\delta_{r, r-i}-\delta_{r, i-1} .
$$

Let us suppose $r+1-i>i$. Then it follows that $\left|L_{r-i}\right|-\left|L_{i-1}\right|=\left|P_{i}\right|$ where

$$
\begin{aligned}
P_{i}=\left\{h \mid h>0, r+h-b_{r+h}-\right. & \sum_{j=r+1-i, r+h-1} \delta_{r+h, j}>0 \text { and } \\
& \left.r+h-b_{r+h}-\sum_{j=i, r+h-1} \delta_{r+h, j} \leqslant 0\right\} .
\end{aligned}
$$

Denoting by $i_{r+h}$ the least positive integer such that $\delta_{r+h, i_{r+h}}=1$, we have $i-1<$ $i_{r+h} \leqslant r-i$, that is, $i-1<i_{r+h}$ and $i-1 \leqslant r-i_{r+h}-1$. Moreover, for $h \in P_{i}$ we have $\delta_{r+h, r-1}=1$ and $r+h-b_{r+h}-\sum_{j=r, r+h-1} \delta_{r+h, j}<r-1$, whence $h \in H$. Thus

$$
\left|L_{r-i}\right|-\left|L_{i}\right|=\left|\left\{h \in H \mid i-1<i_{r+h}, i-1 \leqslant r-i_{r+h}-1\right\}\right|=\left|H_{i-1}\right|-\left|\bar{H}_{i-1}\right| .
$$

Since it is immediate that $\left|N_{i-1}\right|-\left|N_{r-i}\right|=0$, it follows that

$$
\alpha_{r+1-i}-\alpha_{i}=r-2 i+1+\left|H_{i-1}\right|-\left|\bar{H}_{i-1}\right|+\delta_{r, r-i}-\delta_{r, i-1} .
$$

In an analogous way we find the same result if $i>r-i+1$. Now, let $i=1$. It follows that

$$
\alpha_{r}-\alpha_{1}=r+\left|L_{r-1}\right|-\left|N_{r-1}\right|+\left|N_{0}\right| \text {. }
$$

Let us compute $\left|L_{r-1}\right|-\left|N_{r-1}\right|+\left|N_{0}\right|$; we have $N_{r-1}-N_{0}=N^{*}$, where

$$
N^{*}=\left\{h \mid h>0,0<r+h-b_{r+h}-\sum_{j=1, r+h-1} \delta_{r+h, j} \leqslant r-1\right\} .
$$

Moreover, $h \in N^{*}$ implies $\delta_{r+h, r-1}=1$, whence

$$
\left|L_{r-1}\right|-\left|N_{r-1}\right|+\left|N_{0}\right|=\mid\left\{h \mid h>0, \delta_{r+h, r-1}=1 \text { and } h \notin N^{*}\right\} \mid \text {. }
$$

But $h \notin N^{*}$ and $\delta_{r+h, r-1}=1$ imply $r+h-b_{r+h}-\sum_{j=1, r+h-1} \delta_{r+h, j}=0$ whence $\left|L_{r-1}\right|-\left|N_{r-1}\right|+\left|N_{0}\right|=|H|$. Therefore, by an examination of (17), (20) and (21) it easily follows that $\alpha_{r-i+1}-\alpha_{i}=c_{i}$ for every $i \neq r-b_{r}$. If $i=r-b_{r}$, the coefficient of $k^{r-b_{r}-1}$ in $\Delta_{m}$ is

$$
\begin{aligned}
\alpha_{b_{r}+1}-\alpha_{r-b_{r}}+\alpha_{r+1}-\alpha_{b_{r}+1}= & -1-|M|+r-2 r+2 b_{r}+1+\left|H_{r-b_{r}-1}\right| \\
& -\left|\bar{H}_{r-b_{r}-1}\right|+1-\delta_{r, r-b_{r}-1}=c_{r-b_{r}}
\end{aligned}
$$

and the statement is proved. 
4. Adaptation to a Computer. These arithmetic methods for the generation of permutations have been shown to be convenient because they allow one to perform the computation of $\Delta_{m}$ and $\Delta^{m}$ in the most convenient base for the computer used; the specific base $k$ intervenes only in the final passage in which the wanted permutation is generated.

Obviously one must see that $\Delta_{m}$ and/or $\Delta^{m}$ are smaller than the maximum available integer of the standard software. In the opposite case one must use a procedure which performs the operations on the integers in multiple precision.

Since $\Delta_{m}$ is smaller than $k^{k-1}$ and since $10^{9}$ is a normally used integer in the scientific languages of programming, permutations of 10 marks can also be generated by this method with the usual instructions.

Dipartimento di Fisica

Università di Milano

Via Celoria, 16

20133 Milano, Italia

1. J. R. Howell, "Generation of permutations by addition,” Math . Comp., v. 16, 1962, pp. 243-244.

2. J. R. Howell, “Algorithm 87: Permutation generator," Comm. $A C M$, v. 5, 1962, p. 209.

3. D. H. Lehmer, Teaching Combinatorial Tricks to a Computer, Proc. Sympos. Appl. Math. Combinatorial Analysis, Vol. 10, Amer. Math. Soc., Providence, R. I., 1960, pp. 179-193.

4. E. S. PAGE \& L. B. Wilson, An Introduction to Computational Combinatorics, Cambridge Univ. Press, 1979.

5. R. Sedgewick, "Permutation generation methods," Comput. Surveys, v. 9, 1977, pp. 137-163. 